\title{
Performance Evaluation of V2I-Based Channel Aware Floating Car Data Transmission via LTE
}

\author{
Christoph Ide, Bjoern Dusza and Christian Wietfeld \\ Communication Networks Institute \\ TU Dortmund University \\ 44227 Dortmund, Germany \\ e-mail: \{Christoph.Ide, Bjoern.Dusza, Christian.Wietfeld $\} @$ tu-dortmund.de
}

\begin{abstract}
The collection of Floating Car Data (FCD) is very important for dynamic traffic forecasts. For this purpose, sensor nodes in cars are supposed to transmit traffic information via Long Term Evolution (LTE). In this paper, we evaluate this data transmission with regard to several Key Performance Indicators (KPIs) for a channel aware transmission. These indicators are the negative impact on human communication, the power consumption of the mobile devices and the local distribution of cars sending FCD in the scenario. As methodology for performance evaluation a close-to-reality parameterized Markovian model, laboratory data rate as well as power consumption measurements and ray tracing simulations are used. By applying channel aware transmission the Quality of Service (QoS) level of human communication can be obtained and simultaneously the power consumption is significantly reduced. In addition, the fraction of active FCD devices on the highway can be increased.
\end{abstract}

\section{INTRODUCTION}

Highly precise and real time traffic forecasts became a major research topic in the last years. Hereby, the precision of prognosis depends on the quantity, quality and reliability of available information on the traffic flow. The communication aspects of such approaches are as important as the actual prognosis. Moreover, the characteristics of Vehicle-toInfrastructure (V2I) communication as part of Machine-toMachine (M2M) communication is different to conventional LTE Human-to-Human $(\mathrm{H} 2 \mathrm{H})$ communication [1], in relation to the transmitted payload and data rate. The dynamic traffic forecast is based on FCD which contain vehicular sensor data. This data can be transmitted from sensing cars to a server via public cellular communications systems such as for example LTE. Thereby, the negative impact on the existing human communication has to be as small as possible. To ensure this, we use a Channel Sensitive Transmission (CST) for the FCD [2]. The goal in this paper is to analyze the performance of this approach regarding different Key Performance Indicators (KPIs). For this purpose, we have to evaluate the performance of LTE for heterogeneous traffic. In Fig. 1 a system overview for performance analysis of channel sensitive transmission including methodology for the evaluation of the different KPIs is illustrated.

The performance of wireless communication systems can be studied at different abstraction levels: Field trials are necessary to understand the system under realistic conditions. By discrete-event simulations an evaluation of many different configurations is possible. Furthermore, mathematical models provide general insights and elegant solution methods. For performance evaluation regarding the number of servable human connections we use a close-to-reality parameterized Markovian model. The parameterization is based on LTE uplink data rate measurements performed in the laboratory. The model describes the utilization of shared RBs between $\mathrm{H} 2 \mathrm{H}$ and $\mathrm{M} 2 \mathrm{M}$ communication. Hence, the influence of the FCD transmission on the human communication can be analyzed. Furthermore, the power consumption of the LTE User Equipment (UE) is evaluated by measurements in the laboratory and the distribution of active FCD users is evaluated by ray tracing simulations.

The paper is structured as follows: Sec. II gives an overview about the related work. The channel sensitive transmission scheme is described in Sec. III. In Sec. IV the relevant KPIs for FCD transmission are presented and Sec. V illustrates the developed methodology. After that, in Sec. VI the results regarding different KPIs are presented and finally Sec. VII concludes this paper.

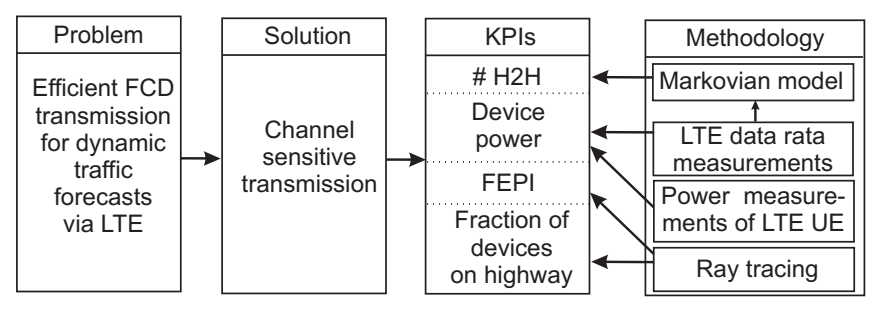

Fig. 1: System overview for performance evaluation of channel sensitive transmission

\section{RELATED WORK}

In [3], FCD is used for the traffic state identification. Thereby, the impact of a small penetration rate of vehicles sending FCD for the reconstruction of the actual travel times is analyzed. In addition, the use of a micro simulation for FCD and its influence on wireless communication systems is shown in [4].

The inclusion of M2M communication into common traffic of cellular communication systems is one of the main goals in the standardization process of LTE-Advanced [1] [5]. In this context, the impact of hundreds of M2M devices on the QoS of $\mathrm{H} 2 \mathrm{H}$ communication should be as small 
as possible. Strategies to keep the complexity of M2M application on different layers as small as possible are shown in [6].

A multi-class Erlang loss model for Orthogonal Frequency-Division Multiplexing (OFDM) systems in [7] is introduced. Every state in the model represents one subcarrier. This Markovian model is adapted in this paper. To make it even more practice-oriented for LTE systems we used the Resource Blocks (RBs) as states. Furthermore, we parameterize the model based on measurements.

Modeling the power consumption of modern smart-phones is gaining importance during the last few years. This comes along with the fact that the power consumption is significantly increasing. In [8], an approach is presented which allows for the automated construction of energy models for Android based smart phones. Instead of measuring the actual current drain from the battery the method described here bases on the battery voltage sensor and knowledge about the battery discharge voltage curve.

A much more detailed investigation of the power consumption of GSM, UMTS and WiFi has been performed in [9]. Here, the impact of different locations and different times are taken into consideration as well as user mobility. In [10] measurements regarding the power consumption of USB enabled LTE UEs are presented. The measurements show that a major non-linearity in the relationship between transmit power and consumed power is caused by a mode transition from low power mode to high power mode of the installed power amplifier. This effect causes to power saving of the channel sensitive transmission.

\section{Channel Sensitive Transmission (CST) SCHEME}

By applying the channel sensitive transmission scheme [2] to the FCD transmission, many M2M devices with good channel conditions transmit FCD. This is guaranteed by a transmission probability $p_{i, j}$ :

$p_{i, j}=\frac{\left(\frac{S N R_{i}}{S N R_{\max }}\right)^{\alpha} \cdot\left(\frac{v_{\max }}{v_{j}}\right)^{\beta}}{\sum_{l=1}^{N} \sum_{k=1}^{M}\left(\frac{S N R_{l}}{S N R_{\max }}\right)^{\alpha} \cdot\left(\frac{v_{\max }}{v_{k}}\right)^{\beta}}, i=1 \ldots N, j=1 \ldots M$

Here, $p_{i, j}$ is the normalized transmit probability for class $i, j$ and is illustrated as a function of $\alpha$ in Fig. 2. The parameter $i$ separates the different SNRs and $j$ the different velocities for the classes of the Markovian model (cf. Tab. I). The arrival rate for each class is multiplied with $p_{i, j} . S N R_{\max }$ is the SNR for which the highest data rate can be achieved and $v_{\max }$ is the highest velocity in the scenario. A method how to estimate the SNR in a real OFDM system in shown in [11]. The parameters $\alpha$ and $\beta$ control the intensity of the channel sensitive transmission scheme. For the Markovian model, we divided the SNR in $N=3$ parts and the velocity in $M=2$ parts. The number of devices which transmit data should be independent on the coefficient $\alpha$. Therefore, we normalize the transmit probability.

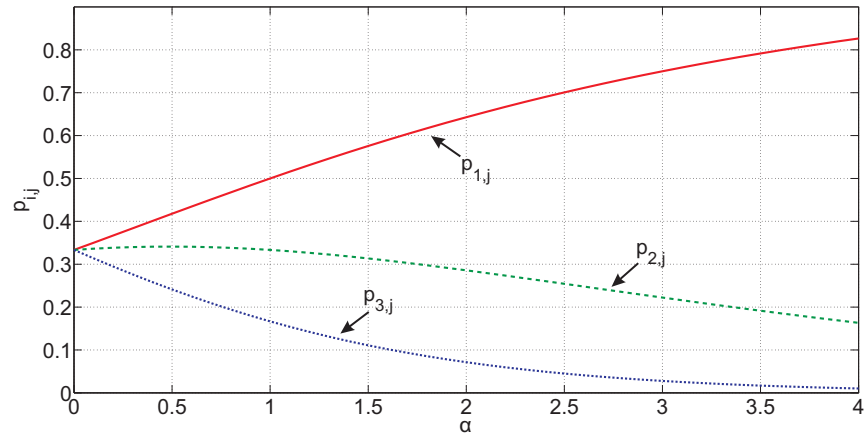

Fig. 2: Transmit probability $p_{i, j}$ for $\beta=0 ; 10 \mathrm{~dB}, 20 \mathrm{~dB}$ and $30 \mathrm{~dB}$ SNR

\section{Key PERFormance IndicATORS (KPIs)}

In this section, the KPIs which are important for the performance analysis of the FCD transmission are described.

- Number of servable H2H users: The transmission of FCD influences the $\mathrm{H} 2 \mathrm{H}$ communication. Thereby, the negative impact should be as small as possible. By means of the Markovian model which is parameterized by laboratory data rate measurements, the number of $\mathrm{H} 2 \mathrm{H}$ users with a certain QoS level is evaluated for different transmission strategies of the FCD. The QoS level is set to $10 \%$ blocking probability and for the FCD transmission we assume that $5 \%$ of all cars transmit FCD. This is a realistic value for a reliable traffic forecast [3].

- Consumed electrical power of the LTE UE. For the transmission of the FCD, common LTE USB sticks or smart-phones can be used. For these devices the power consumption is a major KPI, because the development in battery technology cannot feed the power hungry devices. Therefore, power saving of mobile devices is a relevant topic for the last years. We measured the power consumption in the laboratory and derived an average power for FCD devices.

- FCD Entropy Position Index (FEPI): The distribution of the active FCD users is very important for the dynamic traffic forecast. We introduce the FEPI, which is based on the Theil Index $T$ [12]:

$$
T=\frac{1}{K} \sum_{i=1}^{K}\left(\frac{x_{i}}{\bar{x}} \cdot \ln \frac{x_{i}}{\bar{x}}\right)
$$

Thereby, the scenario is divided into $K$ parts. We used $K=16 . \bar{x}$ is the average number of users per part and $x_{i}$ is the number of users in part $i$. From this value we derived the FEPI:

$$
F E P I=\sqrt{\frac{T_{0}}{T}}
$$

$T_{0}$ is the Theil Index without channel sensitive transmission. This KPI is derived from ray tracing simulations and describes how homogeneously the users are distributed in the scenario. The more homogeneous the 
distribution of the number of users in the parts the higher the FEPI.

- Fraction of FCD vehicles on the highway: For the dynamic traffic forecast FCD from cars which are traveling on the highway are of special interest. This KPI is also gained from ray tracing simulations.

\section{Methodology for Evaluation of the Impact of Channel Sensitive Transmission ON The KPIS}

In this section, the methodology for the evaluation of the KPIs is described. This includes the Markovian model, laboratory measurements and ray tracing simulations.

\section{A. System Model for Evaluation of the Impact of FCD Transmission on Human Communication}

For evaluation of the impact of different transmission strategies on the LTE air interface, a close-to-reality parameterized Markovian model is used. This system model is introduced in [16]. Assigning the user requests to different classes of resources, each class is modeled by one dimension. To represent different channel conditions, the $\mathrm{H} 2 \mathrm{H}$ and M2M communication is splitted up into six classes. These classes are defined by the SNR and by the user velocity. As result, the model calculates the blocking probability of $\mathrm{H} 2 \mathrm{H}$ communication $p_{b_{H}}$ as a function of the arrival rate for M2M $\lambda_{M}$ and the transmission strategy (cf. Fig. 3). The M2M communication is characterized by the FCD payload (mean $100 \mathrm{kByte}$ ). Hence, the duration of transmission respectively the service rate $\mu_{M}$ depends on the data rate, which is a function of the channel conditions that we determined by measurements. In addition, $10 \mathrm{RBs}$ are used in the frequency domain for the FCD transmission $\left(c_{M}=10\right)$. The arrival rate for M2M users is adjustable. It influences the blocking probability of human users and is chosen so that the blocking probability does not exceed $10 \%$. For $\mathrm{H} 2 \mathrm{H}$ communication a video streaming service with a fixed data rate of $250 \mathrm{kbit} / \mathrm{s}$ is assumed. Therefore, the number of RBs $c_{H}$ needed for this data rate and different channel conditions is evaluated by measurements. The service rate $\mu_{H}$ and arrival rate $\lambda_{H}$ are given by the system parameterization. Furthermore, the power consumption of the LTE USB stick for different channel conditions is measured in the laboratory.

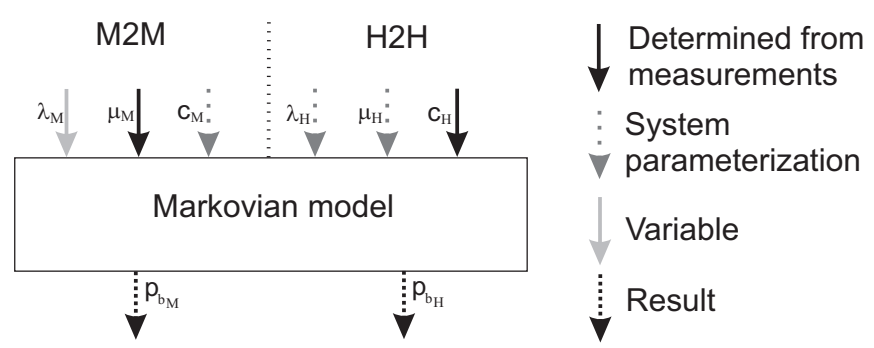

Fig. 3: Markovian model for the evaluation of the influence of FCD transmission on the utilization of the LTE radio interface

\section{B. Laboratory Measurement Setup for Data Rate and Power} Measurements of FCD Devices

The Markovian model is parametrized by LTE data rate measurements. For the evaluation of the User Datagram Protocol (UDP) uplink data rate for different channel conditions laboratory measurements are performed. A base station emulator (R\&S CMW 500) sets up an LTE cell in band 7 (2.62 GHz). Hereby, the modulation and coding schemes with the indexes 0,3 and 6 are used. The base station emulator is connected to a fading channel emulator (Elektrobit Propsim C8) via RF cable (cf. Fig. 4). On this equipment an ITU Vehicular A channel model [13] realistically effects the LTE signal. In addition, an Additive White Gaussian Noise (AWGN) with a fixed SNR can be added to the RF connection. The category 3 LTE User Equipment (UE) Samsung GT B3730 completes the setup. For the UDP data rate measurements the LTE base station and the UE are connected to PCs with iPerf running. More details on the used setup, especially the channel emulator can be found in [14]. In [2] more results from a data rate measurement campaign are shown.

For the determination of the power that is consumed by the USB enabled LTE device, a measurement probe is placed between the actual client PC and the UE. Therefore, the electrical energy powering the LTE stick has to pass the probe where it is sampled at a frequency of $100 \mathrm{kSamples} / \mathrm{s}$. The so derived raw data is transferred to an evaluation PC via USB where the measurement can be analyzed in terms of for example minimum, maximum and average power consumption as well as the integration over individual time intervals for the determination of the consumed energy in this interval. The high resolution of the measurement does furthermore allow for a detailed examination of the power consumption.

\section{Analytical Markovian Model}

For performance evaluation of LTE a multi-class Erlang loss model is used [16]. The analytical Markovian model describes the shared LTE RBs. For the different traffic classes a different number of states is needed and each class is represented by one dimension in this model. The dimension of the model is reduced by an approach presented in [15].

TABLE I: LTE system parametrization

\begin{tabular}{c|c} 
Measurement parameter & Value \\
\hline Carrier frequency & $2.62 \mathrm{GHz}(\mathrm{LTE}$ band 7) \\
\hline Channel bandwidth & $10 \mathrm{MHz}(50 \mathrm{RBs})$ \\
\hline RBs for FCD transm. & 10 \\
\hline FCD payload (mean) & $100 \mathrm{kByte}$ \\
\hline FCD transm. frequency & $f=0.1 / \mathrm{s}$ \\
\hline Duplexing scheme & FDD \\
\hline Number of classes & $N=3, N=2$ \\
\hline Fading channel model & ITU Vehicular A \\
\hline SNR for classes $i$ & 10,20 and $30 \mathrm{~dB}$ \\
\hline Velocity for classes $j$ & 60 and $120 \mathrm{~km} / \mathrm{h}$ \\
\hline$S N R_{\max }$ for CST & $40 \mathrm{~dB}$ \\
\hline$v_{\max \text { for CST }}$ & $150 \mathrm{~km} / \mathrm{h}$ \\
\hline UE & Samsung GT B3730 \\
\hline RLC mode & Acknowledged mode
\end{tabular}




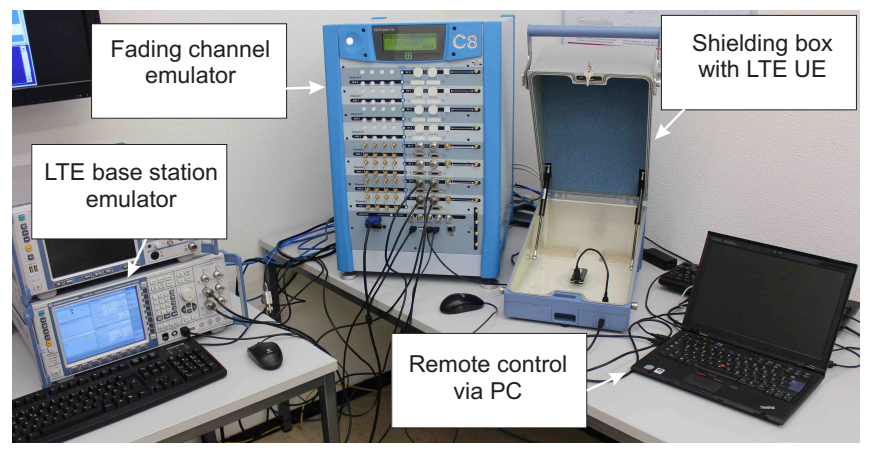

Fig. 4: Measurement setup for LTE data rate and power measurements for LTE USB Sticks and smart-phones

Therefore, a one dimensional model with a different number of states per user can be developed. The result of the model is the blocking probability (cf. [16]) which can be calculated for the assumption that the inter-arrival time for the arrival rate $\lambda_{s}$ and services rate $\mu_{s}$ for every class $s$ follows a negative exponential distribution.

The service rate for the $\mathrm{H} 2 \mathrm{H}$ communication is set to 1 per second and the arrival rate to 4 per second. The service rate for the V2I users is calculated as data rate, which is taken from the laboratory measurements, divided by the payload of transmitted FCD. For more details about the classes and the parametrization of the model, we refer to [16].

\section{Modeling the Power Consumption of M2M Devices for the FCD Transmission}

We model the average power consumption for a FCD device $P_{F C D}$ by three parts: The power for the listening mode $P_{L}$, the energy for one random access $E_{R A, i, j}$ divided by the random access time and the power for the actual transmission $P_{i, j}$ for the class $i, j$. The time for the transmission can be calculated by dividing the payload $D$ by the data rate $D R_{i, j}$. $f$ is the FCD transmission frequency. $P_{L}, E_{R A, i, j}, P_{i, j}$ and $D R_{i, j}$ are measured in the laboratory (cf. Tab. II and Fig. 6).

$P_{F C D}=P_{L}+f \cdot \sum_{i=1}^{N} \sum_{j=1}^{M}\left(p_{i, j} \cdot\left(E_{R A, i, j}+\frac{D}{D R_{i, j}} \cdot P_{i, j}\right)\right)$

\section{E. Estimation of the Local Distribution of FCD Devices by Ray Tracing Simulations}

To evaluate the local distribution of FCD devices, we use a realistic channel model with a 3D Intelligent Ray Tracing (AWE Probsim [17]) and a detailed 3D CAD model of an urban scenario. This enables an accurate determination of the SNR. As propagation class double reflection and single diffraction is used. The Base Station (BS) antenna has an opening angle of $120^{\circ}$ with $16.7 \mathrm{~dB}$ gain and a downtilt of $3^{\circ}$. Furthermore, the UE antenna gain is $1 \mathrm{dBi}$, the UE noise figure is set to $6 \mathrm{~dB}$ and the transmission power of the $\mathrm{UE}$ is $23 \mathrm{dBm}$.

\section{RESUlts}

The presentation of the results is organized as follows: First the laboratory measurement results regarding data rates and power consumption for different channel conditions are shown. With these information, the average power of the LTE UE for different values of $\alpha$ is analyzed in the next step. Furthermore, the impact of channel sensitive transmission on the distribution of users in the scenario and the fraction of users on the highway is presented. Finally, the results are summarized by an investigation of all KPIs.

\section{A. Laboratory Measurements of LTE Data Rates and Power Consumption}

The power consumption for the random access procedure can be divided into three parts (cf. Fig. 5). First the RF component of the USB stick is disabled and the Radio Resource Control (RRC) is in listening state. Then, the random access procedure takes place. It can be seen from the plot that a random access preamble is sent many times with a increasing transmission power $P_{T x}$. This results in an increasing power consumption of the LTE UE. After a successful random access the RRC gets into the connection state and the data transmission starts. We measured the consumed power of the random access procedure for different channel condition (cf. Tab. II). This energy is needed before every FCD transmission.

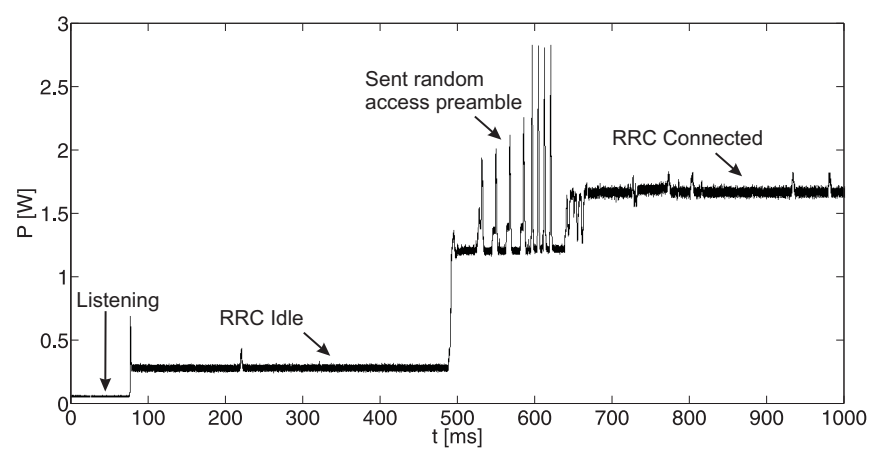

Fig. 5: Measured power consumption of LTE UE during random access procedure

Furthermore, we measured the LTE uplink data rate and the power consumption of the LTE UE for different channel conditions in the laboratory for the other states of our energy model. In Fig. 6 the data rate and the corresponding consumed power $P_{i, j}$ for the transmission and listening mode for different SNRs is presented. Thereby, fractional path loss compensation [18] with a compensation factor of 0.5 and $10 \mathrm{RBs}$ per user are assumed. One can see from the figure that the data rate decreases significantly for a lower SNR.

TABLE II: Measured energy for random access

\begin{tabular}{c|c|c|c|c}
$\mathbf{i}$ & $\mathbf{j}$ & $\mathbf{S N R}$ & $P_{T x}$ & $E_{R A, i, j}$ \\
\hline 1 & 1,2 & $30 \mathrm{~dB}$ & $-7 \mathrm{dBm}$ & $0.28 \mathbf{J}$ \\
\hline 2 & 1,2 & $20 \mathrm{~dB}$ & $-2 \mathrm{dBm}$ & $0.32 \mathrm{~J}$ \\
\hline 3 & 1,2 & $10 \mathrm{~dB}$ & $+3 \mathrm{dBm}$ & $0.36 \mathbf{J}$
\end{tabular}


In contrast to that, the consumed power increases for a lower SNR, because a higher transmission power has to be used. In [10], it is shown how the consumed power of the used UE depends on the transmission power. For a very high transmission power the UE enables a high power mode and consumes overproportionally more power. Hence, the energy per bit increases significantly for worse channel conditions. The velocity has no major impact on the consumed power. For the transmission of FCD this means that it needs much more power if the channel conditions are bad. Furthermore, the transmission is much faster for good conditions and the LTE UE can therefore return faster to the energy saving listening mode. In this state, the LTE UE consumes only $45 \mathrm{~mW}$ (cf. Fig. 6).

Therefore, the channel sensitive transmission saves power. In Fig. 8 one can see the average power for differently parameterized channel sensitive transmission schemes. The listening power and the power for the random access need about $20 \%$ of the total power. By applying the presented channel sensitive transmission scheme with $\alpha=2$ and $\beta=2,32 \%$ of the total power can be saved. If one regards only the power for the actual data transmission, the power can be reduced by up to $42 \%$.

\section{B. Distribution of Active Users in the Urban Scenario}

The collection of FCD is the basis for a dynamic traffic forecast. For this purpose, it is important which cars transmit FCD. The data is of special importance from cars which are traveling on a highway. Furthermore, the FCD should

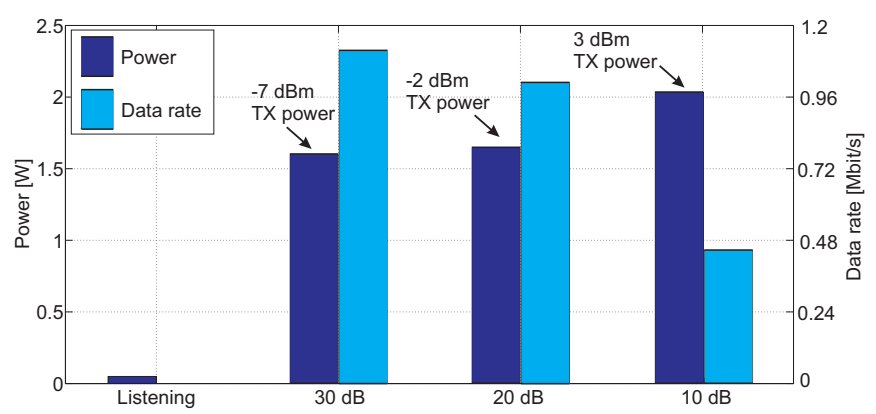

Fig. 6: Trade-off between consumed power and data rate for different states for 10 RBs per user

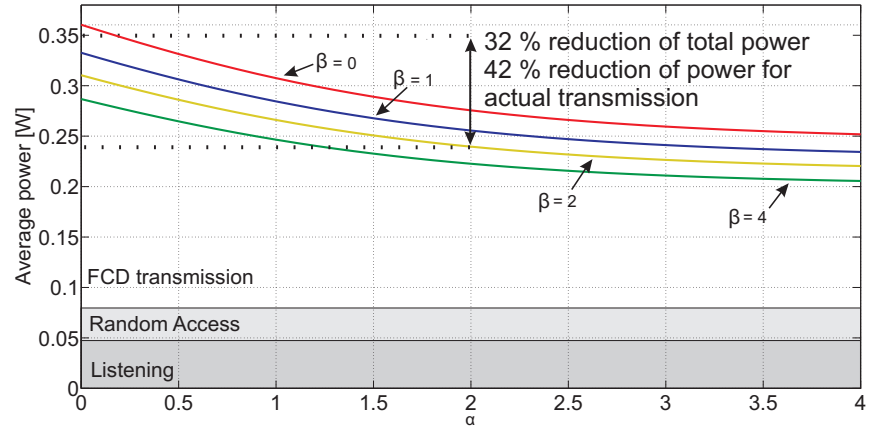

Fig. 8: Average power consumption of LTE FCD devices for channel sensitive transmission with $\alpha[0 ; 4]$ and $\beta[0 ; 4]$

be collected from cars with different positions. It is not helpful for the dynamic traffic forecast, if only vehicles very close to the base station send data. Hence, we use two KPI for the performance evaluation of the distribution of cars in the scenario. On the one hand site, the FEPI which describes how homogeneously the users are distributed in the area. On the over hand site, the fractions of cars which sends FCD and are traveling on the highway. In Fig. 7 one can see the distribution of vehicles for differently intensive channel sensitive transmission schemes. These results are taken from the ray tracing simulation. We divided the users into different groups based on their SNR (cf. Tab. I). If no channel sensitive transmission scheme is used, the users are uniformly distributed, because the transmission probability is independent of the SNR and the number of users per group is the same. For a higher value of $\alpha$, FCD is transmitted very often for good channel conditions. Therefore, many users with a high SNR send data. Although at first sight this seems to have a negative impact on the dynamic traffic forecast, one has to analyze in which areas the channel conditions are good. If we have a look on Fig. 7d, we see that many users on the highway are active. This is due to the position of the Base Station (BS). We used a very common position directly beside the highway for the ray tracing investigation. Hence, many positions on the highway are very well connected. This means that the FEPI decreases by applying channel sensitive transmission, but the fraction of active FCD devices in the area of special interest increases.

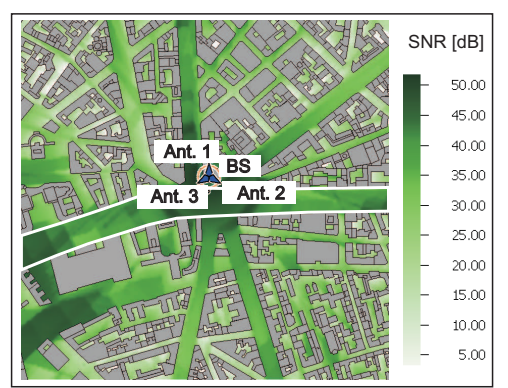

(a) Ray tracing results

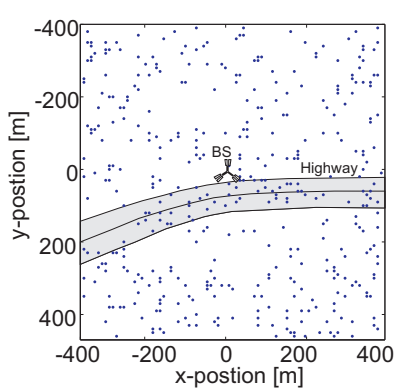

(b) $\alpha=0 \rightarrow F E P I=1$

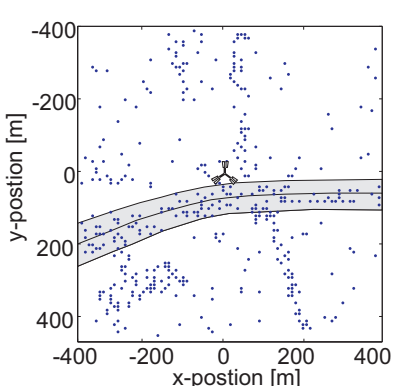

(c) $\alpha=2 \rightarrow F E P I=0.40$

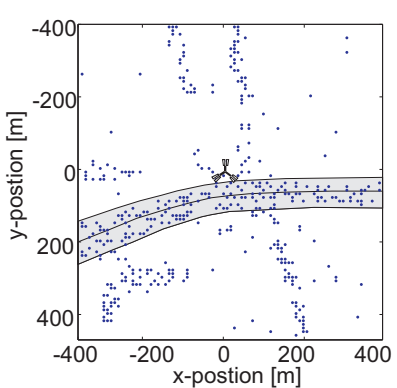

(d) $\alpha=4 \rightarrow F E P I=0.30$

Fig. 7: User positions for channel sensitive FCD transmission with parameter $\alpha[0 ; 4]$ and $\beta=0$ 


\section{Overview of KPIs for Channel Sensitive Transmission}

An overview of the KPIs for channel sensitive transmission is given in Fig. 9. For making the results comparable, the values for each KPI are normalized by the KPI values for $\alpha=0$, which represent a transmission without channel awareness. It can be seen from the figure that the number of $\mathrm{H} 2 \mathrm{H}$ connections increases by applying the scheme. For $\alpha=4$ the number of $\mathrm{H} 2 \mathrm{H}$ connections is $75 \%$ higher in relationchip to $\alpha=0$. This result is taken from the Markovian model. The average power consumption decreases by $35 \%$. This means that the negative impact of the FCD transmission on the $\mathrm{H} 2 \mathrm{H}$ communication decreases although the FCD devices save power. The cost for this gain is the smaller FEPI. The devices transmit FCD dependent on the channel conditions. Hence, the distribution of the users gets organized. This drawback is not that critical for the dynamic traffic forcast, because the number of cars which are on the highway and transmit FCD increases by over $150 \%$.

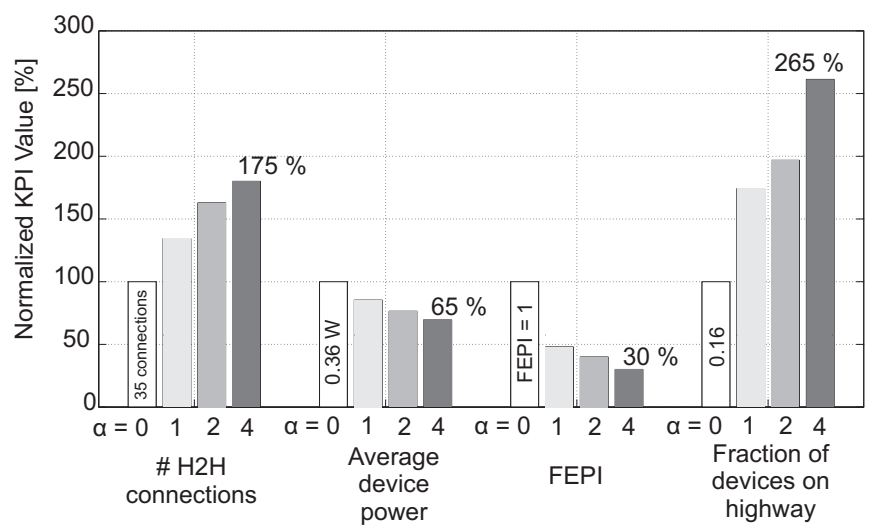

Fig. 9: KPIs for channel sensitive FCD transmission with parameter $\alpha[0 ; 4]$ and $\beta=0$

\section{CONCLUSION}

In this paper, we evaluated the FCD collection for different transmission strategies. For this purpose, KPIs were defined including the power consumption of the LTE UE, the distribution of FCD devices in the scenario and the negative impact of FCD transmission on human-to-human communication. For modeling the traffic of an LTE cell a closeto-reality parameterized Markovian model was used. The evaluation of the power consumption is based on laboratory measurements using a real LTE UE and a standard conform LTE link to a base station emulator. The distribution of FCD users is investigated by ray tracing simulations. The results show that the channel sensitive transmission scheme significantly reduces the negative impact of FCD transmission on the $\mathrm{H} 2 \mathrm{H}$ communication and the power consumption of the LTE UE for FCD transmission. Although, the distribution of active FCD devices gets inhomogeneous by applying the channel sensitive transmission scheme, the fraction of cars on the highway sending FCD can be more than doubled.

\section{ACKNOWLEDGMENT}

Part of the work on this paper has been supported by Deutsche Forschungsgemeinschaft (DFG) within the Collaborative Research Center SFB 876 "Providing Information by Resource-Constrained Analysis", projects B4 and A4. Our work has been partially funded by the SPIDER project, which is part of the nationwide security research program funded by the German Federal Ministry of Education and Research (BMBF) (13N10238).

\section{REFERENCES}

[1] 3GPP TS 22.368, Service Requirements for Machine-Type Communications, version 13.3

[2] C. Ide, B. Dusza, M. Putzke and C. Wietfeld, Channel Sensitive Transmission Scheme for V2I-based Floating Car Data Collection via LTE, IEEE International Conference on Communications (ICC) Workshop on Intelligent Vehicular Networking: V2V/V2I Communications and Applications, Ottawa, Canada, Jun. 2012

[3] B. S. Kerner, C. Demir, R. G. Herrtwich, S. L. Klenov, H. Rehborn, M. Aleksi, A. Haug, Traffic state detection with floating car data in road networks, 8th International IEEE Conference on Intelligent Transportation Systems, Vienna, Austria, Sep. 2005

[4] B. S. Kerner, S. L. Klenov and A. Brakemeier, Testbed for Wireless Vehicle Communication: a Simulation Approach based on Three-Phase Traffic Theory IEEE Intelligent Vehicles Symposium, Eindhoven, Netherlands, Jun. 2008

[5] 3GPP TR 23.888, System Improvements for Machine-Type Communications, version 1.2

[6] Y. Chen and W. Wang, Machine-to-Machine Communication in LTE-A, 72nd IEEE Vehicular Technology Conference, Ottawa, Canada, 2010

[7] V. Pla, J. Martinez-Bauset and V. Casares-Giner, Comments on "Call Blocking Probability and Bandwidth Utilization of OFDM Subcarrier Allocation in Next-generation Wireless Networks", Communications Letters, vol. 12, no. 5, May 2008

[8] L. Zhang, B. Tiwana, Z. Qian, Z. Wang, R. Dick, Z. Mao and L. Yang, Accurate Online Power Estimation and Automatic Battery Behavior Based Power Model Generation for Smartphones, Eighth IEEE/ACM/IFIP International Conference on Hardware/Software Codesign and System Synthesis, Grenoble, France, Oct. 2010

[9] N. Balasubramanian, A. Balasubramanian, and A. Venkataramani, Energy Consumption in Mobile Phones: A Measurement Study and Implications for Network Applications, 9th ACM SIGCOMM conference on Internet measurement, Barcelona, Spain, Aug. 2009

[10] B. Dusza, C. Ide and C. Wietfeld, Utilizing Unused Network Capacity for Battery Lifetime Extension of LTE Devices, IEEE International Conference on Communications (ICC) Workshop on Novel Approaches to Energy Measurement and Evaluation in Wireless Networks (NovaEnEv), Ottawa, Canada, Jun 2012

[11] S. A. Kim, D. G. An, H.-G. Ryu, J.-U. Kim, Efficient SNR estimation in OFDM system, IEEE Radio and Wireless Symposium (RWS), Phoenix, USA, 2009

[12] H. Theil, Principles of Econometrics, Wiley, 1971

[13] International Telecommunication Union, Recommendation ITU-R M.1225 Guidelines for Evaluation of Radio Transmission Technologies for IMT-2000, 1997

[14] C. Ide, B. Dusza and C. Wietfeld, Mobile WiMAX Performance Measurements with Focus on Different QoS Targets, 18th IEEE Workshop on Local and Metropolitan Area Networks (LANMAN), Chapel Hill, North Carolina, USA, Oct. 2011

[15] J. S. Kaufmann, Blocking in a Shared Resource Environment, IEEE Transactions on communications, vol. 29, no. 10, October 1981

[16] C. Ide, B. Dusza, M. Putzke, C. Mueller and C. Wietfeld, Influence of M2M Communication on the Physical Resource Utilization of LTE, 11th Wireless Telecommunications Symposium, London, UK, Apr 2012

[17] G. Woelfle, R. Hoppe, and F. M. Landstorfer, A Fast and Enhanced Ray Optical Propagation Model for Indoor and Urban Scenarios, Based on an Intelligent Preprocessing of the Database, 10th IEEE International Symposium on Personal, Indoor and Mobile Radio Communications, Osaka, Japan, Sep., 1999

[18] 3GPP, TS 36.213, LTE Physical Layer Procedures, V 9.3.0, Sep. 2009 